\title{
e-Phaïstos
}

e-Phaïstos

Revue d'histoire des techniques / Journal of the history

of technology

I-2 | 2012

Les sources de l'histoire des techniques

\section{Lire les images de machines. Essai pour une typologie analytique des images techniques (XVe- début XIXe)}

Reading pictures of machines. Essay for an analytical typology of technical pictures (15th - early 19th century)

\section{Benjamin Ravier-Mazzocco}

\section{OpenEdition}

\section{Journals}

Édition électronique

URL : http://journals.openedition.org/ephaistos/450

DOI : 10.4000/ephaistos.450

ISSN : 2552-0741

Éditeur

IHMC - Institut d'histoire moderne et contemporaine (UMR 8066)

Édition imprimée

Date de publication : 1 décembre 2012

Pagination : $54-68$

ISSN : 2262-7340

\section{Référence électronique}

Benjamin Ravier-Mazzocco, "Lire les images de machines. Essai pour une typologie analytique des images techniques (XVe-début XIXe) », e-Phaïstos [En ligne], I-2 | 2012, mis en ligne le 09 septembre 2016, consulté le 10 mai 2020. URL : http://journals.openedition.org/ephaistos/450 ; DOI : https:// doi.org/10.4000/ephaistos. 450 


\title{
Lire les images de machines Essai pour une typologie analytique des images techniques ( $X V^{e}$-début $X I X^{e}$ )
}

\author{
Benjamin Ravier-Mazzocco \\ doctorant au Centre d'Histoire des \\ Techniques (CH2ST/EA 127), \\ Université Paris 1 Panthéon-Sorbonne
}

\begin{abstract}
Introduction
L'analyse historique des images s'est beaucoup développée ces dernières années, et les images de machines n'ont pas échappé à cette vague de fond. Deux approches ont été privilégiées : d'une part la compréhension des conditions et finalités sociales de la création des images, d'autre part la mise en lumière de la progressive émergence d'un graphisme technique tel qu'on le connaît aujourd'hui ${ }^{1}$. L'articulation de ces deux démarches est cependant très difficile, notamment parce qu'il semble que les techniques graphiques utilisées ne dépendent pas exclusivement des objectifs attribués aux différentes images. Serait-il alors impossible de faire le lien entre l'environnement social dans lequel sont produites les images et leurs modes de représentation ? La principale difficulté, à mon sens, est le fait qu'on ne puisse pas penser ces fonctions cumulées dans une même image. Sans doute la typologie utilisée, pour éclairante qu'elle soit, est un peu trop restrictive. La nécessité d'utiliser des catégories plus générales, et surtout potentiellement cumulatives a guidé cet essai. Les théâtres de machines, et, de façon élargie, les images de machines de l'époque moderne
\end{abstract}

et pré-moderne (XIV ${ }^{\mathrm{e}} \mathrm{XV} \mathrm{V}^{\mathrm{e}}$ siècle), offrent ici un terrain de choix, notamment parce que les livres eux-mêmes confessent plusieurs objectifs : éduquer le prince, obtenir de lui protection, asseoir la paternité de l'auteur sur ses inventions, promouvoir son génie créatif, instruire le lecteur sur la mécanique. Par ailleurs, ils couvrent une assez longue période (jusqu'au XVIII ${ }^{\text {e siècle) }}$ et utilisent des techniques graphiques très diverses. C'est pourquoi, même si les catégories proposées se veulent valables pour d'autres types d'images représentant les techniques, les exemples utilisés ici sont tirés des images de machines, et notamment des théâtres de machines de l'époque moderne ${ }^{2}$.

On proposera d'attribuer au moins quatre fonctions principales aux images : témoigner ou rendre compte ; expliquer ; concevoir ; et reproduire. Cette étude se focalise sur les deux premières et tente de montrer la façon dont ces fonctions influencent les modes de représentation des machines. Une dernière partie évoquera les deux dernières fonctions définies, notamment en ce qu'elles doivent aux différents outils développés pour répondre aux exigences du témoignage et de l'explication. 


\section{La fonction testimoniale}

Les théâtres de machines, notamment ceux rédigés par des ingénieurs français, ont sans conteste un objectif courtisan, voire carriériste : il s'agit d'obtenir un poste de la part d'un prince. Les ingénieurs doivent assurément séduire par l'image, mais surtout témoigner de leur inventivité, et cela en montrant leurs inventions. Montrer, c'est d'abord rendre compte visuellement de quelque chose, fut-ce imaginaire. Les auteurs des traités de réduction en art qui décrivent les gestes et les outils des métiers ne font pas autre chose : ils témoignent de l'existence et de l'usage de quelques machines et instruments. Ils témoignent et ils enregistrent, c'est-à-dire qu'ils permettent à la scène représentée - saisie sur le motif, inventée ou recopiée d'un manuscrit - d'être conservée sous la forme d'une reproduction graphique en deux dimensions. Ces fonctions de témoignage et d'enregistrement sont primordiales, et si la photographie, et plus récemment l'infographie, ont sans doute pris aujourd'hui une grande part de cette fonction, elle n’a jamais totalement disparu.

\section{Une machine mise en scène.}

L'objectif n'est ni de comprendre ni d'expliquer, ni même de reproduire, mais simplement de montrer que la chose est, ou, à la rigueur, puisse être. De ce fait, la fonction de témoignage conduit l'auteur de l'image à se rapprocher d'un mode réaliste de description visuelle, qui pousse à l'utilisation si répandue, et aujourd'hui si troublante quand elle concerne les machines, de la perspective. Il s'agit de coller à l'expérience visuelle, la vue prenant une place prépondérante dans la hiérarchie des sens dans l'Europe de la Renaissance, et d'autant plus que se met en place une éthique de l'expérimentation. Steven Shapin a ainsi très bien montré que les comptesrendus d'expérience de Robert Boyle accompagnaient des descriptions très circonstanciées et détaillées d'images précises : « ce n'est pas le dessin d'une «idée » de pompe, mais celui d'une pompe réelle. $»^{3}$.

Il serait possible de faire la même remarque concernant toutes les machines des théâtres de machines. Cela éclaire du même coup l'intérêt de la mise en scène de la machine. Si l'intérêt de la mécanique réside, comme le prétendent les auteurs, dans l'apport économique que le mouvement de celle-ci génère, il est logique de représenter la machine dans son cadre de fonctionnement, la machine gagnant à être représentée in situ et en perspective. Une autre explication, empruntée au concept de "bibliothèque de situation ", avancé par Anne-Françoise Garçon ${ }^{4}$, résiderait dans le fait que montrer la machine dans un environnement familier contribue à rendre acceptable, normale, l'innovation dont elle est porteuse. Le témoignage vient ici ancrer dans un réel déjà existant une nouveauté perçue comme perturbatrice; en habituant l'œil du lecteur, l'image ici neutralise le risque de l'innovation.

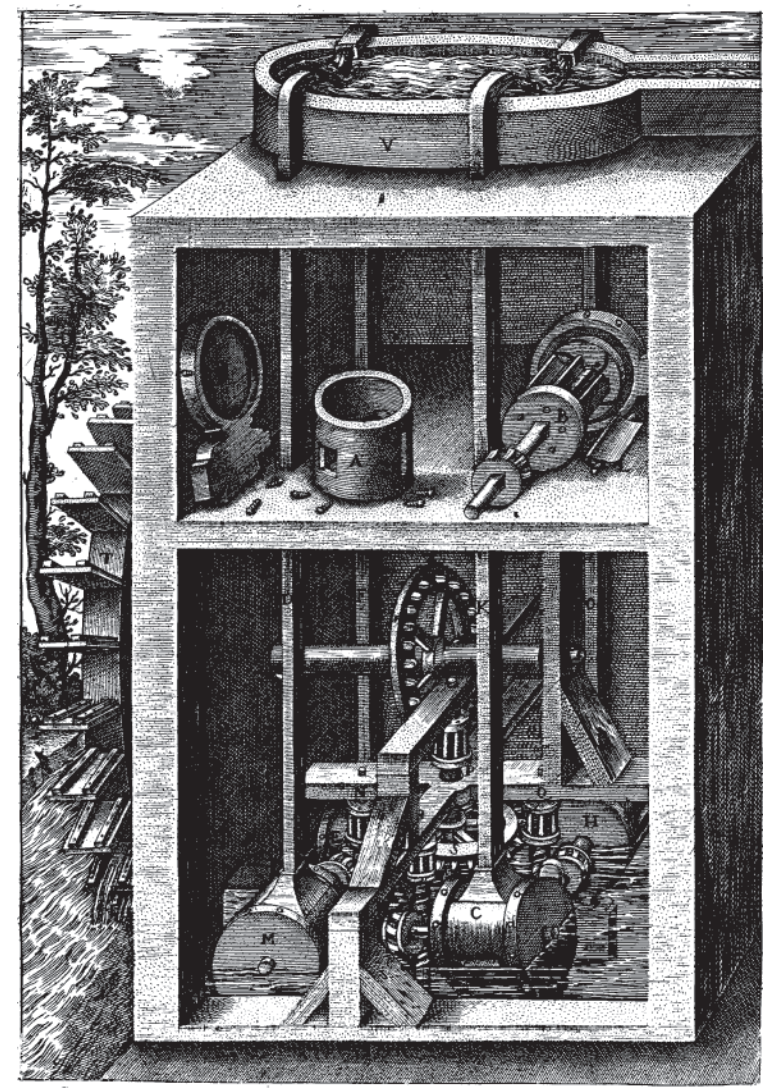

Fig. 1 : Planche 51 de RAMELLI Agostino, Le diverse et artificiose machine, Paris, chez l'auteur, 1588 (Conservatoire national des arts et métiers, Conservatoire numérique http://cnum.cnam.fr). 
Cette mise en situation de la machine comporte ainsi un certain nombre d'informations visuelles minimales que nous retrouvons aussi bien dans les carnets personnels de Villard de Honnecourt (XIII ${ }^{\mathrm{e}} \mathrm{s}$.), Léonard de Vinci (XV-XVI ${ }^{\mathrm{e}} \mathrm{s}$.) ou Heinrich Schickhardt (XVI-XVII ${ }^{\mathrm{s}} \mathrm{s}$ ), que dans les traités de mécanique et les livres de machines, manuscrits ou imprimés. Ainsi, le bâti de la machine, dut-il être réduit à sa plus simple expression, est toujours représenté, ancrant visuellement les mécanismes dans une spatialité, qui témoigne de la réalité de la machine pour le lecteur autant qu'elle aide l'inventeur à penser la disposition des organes mécaniques.

Forces mouvantes fréquentes, l'eau et le vent sont aussi représentés sur le dessin quand ils interagissent avec la machine, ne serait-ce que sous la forme d'un jet d'eau ou de quelques traits ondulés, marquant la direction du vent. De même, si les chevaux, les ânes ou les bœufs ne sont pas toujours montrés, sans doute en raison du manque de temps ou du coût supplémentaire qu'engageraient ces mentions, toutes les machines qui utilisent la force animale sont représentées, non seulement avec le manège, mais aussi avec le harnais ou le joug nécessaires à l'attelage. Une disparité similaire, a priori pour les mêmes raisons, s'observe dans la représentation des humains au travail dont l'auteur se dispense parfois quand le mode d'entraînement de la machine est évident, comme c'est le cas des instruments scientifiques ou des manivelles.

Aux forces mouvantes et au bâti, il faut ajouter aussi le résultat, qui est souvent représenté, qu'il s'agisse d'un objet fini ou de l'adduction d'eau. À défaut de montrer le mouvement, les images cherchent ainsi à le suggérer. Il arrive même que certains objets disposés autour de la machine permettent de rendre compte d'outils interchangeables, et proposent donc de témoigner des possibilités de personnalisation - de « customisation » dirait-on aujourd'hui - de la machine.

À ces informations visuelles de base peuvent s'ajouter ensuite d'autres représentations permettant de compléter la contextualisation de la machine. Le lieu d'utilisation de la machine est ainsi souvent suggéré, pour ne pas dire parfois représenté, que ce soit un atelier, un camp militaire, une mine ou les abords d'un fleuve, qu'on le trouve en ville, dans un village ou en rase campagne. De la même façon, certains auteurs ajoutent à la mise en situation de la machine différents personnages en plus des ouvriers servant de force motrice. L'usager est souvent représenté, qu'il s'agisse d'un maître artisan travaillant sur son tour, ou d'un bourgeois transporté dans un véhicule particulier. Parfois, les auteurs représentent un riche seigneur en visite, empruntant ici certains codes de la peinture et de la tapisserie ${ }^{5}$. Rarement cependant la foule ou un public quelconque n'est représenté, sauf dans le cas de machines aux effets particulièrement extraordinaires. On peut donc dire que si les images destinées à un public mettent davantage les machines en situation, la représentation obéit, de façon générale, aux codes d'un réalisme qu'exige la fonction testimoniale de l'image technique. Notons que, sur ce point, les théâtres de machines n'ont fait que généraliser des techniques graphiques déjà existantes, et ne se présentent pas en rupture avec d'autres traditions manuscrites ou imprimées.

\section{Montrer jusqu'aux entrailles de la machine}

$\mathrm{Si}$ le réalisme de la fonction testimoniale de l'image pousse à la comparaison avec la photographie, il ne faut pas croire pour autant que la technique du dessin, et celles qui lui sont afférentes (la gravure sur bois et la gravure sur cuivre notamment) obéissent aux mêmes règles. Le coût et le temps de la création de l'image nécessitent en effet, pour montrer une même quantité d'information, de condenser dans une image ce qu'on montrerait, avec la photographie, en plusieurs images. D'une certaine manière, l'infographie, et les images , fixes ou vidéos, que l'on trouve dans les ouvrages de vulgarisation ou dans les musées, permettant de montrer l'intérieur et le fonctionnement de machines ou 
d'objets présentés par différentes techniques de transparence, de superposition de couches, ou de schématisation, sont plus proches, par les codes graphiques qu'elle utilise, de cette iconographie technique. En effet, témoigner du bon fonctionnement d'une machine ou de tout autre métier suppose d'en montrer des facettes que ne pourrait pas toujours montrer une vue globale, extérieure.

Pour cela, les auteurs utilisent ce qu'on peut appeler des techniques de dévoilement. Il ne s'agit pas ici de techniques visant à montrer ce qu'un visiteur ne pourrait pas voir, ni de dévoiler un secret quelconque. Il s'agit au contraire de montrer en une seule fois ce qui nécessiterait plusieurs vues, et pour cela, il faut littéralement ouvrir les portes, couper les murs et souvent réorganiser l'espace de l'atelier pour y donner à voir, quand cela est nécessaire, ce que des obstacles visuels cacheraient. La plus répandue des techniques graphiques utilisées est sans doute celle, bien connue, de la coupe, mais d'autres techniques sont utilisées comme la vue par transparence ou encore ce que Markus Popplow et Wolfgang Lefèvre appellent la « cutaway view » : il s'agit de couper ou de retirer quelques petites parties du bâti, d'un mur ou du sol, juste au niveau de la transmission d'un engrenage à une lanterne, de manière à permettre au lecteur de bien saisir cette transmission. Une technique qui s'accompagne le plus souvent de la représentation d'un bâti « aérien », c'està-dire limité à la seule représentation des poutres, comme on le ferait sur un modèle, sans représenter les murs de l'atelier ou la toiture.

La fonction de témoignage amène parfois à réorganiser l'espace de manière à y placer plus d'informations. Prenons l'exemple de la représentation du papetier par Jost Amman ${ }^{6}$. La représentation des roues à eau à l'arrière de l'image est en évidente contradiction avec le sens de l'arbre à cames visible au second plan. Il est clair qu'il s'agit ici d'un procédé volontaire à des fins presque pédagogiques : le but de l'image est de montrer ce qui se rapporte au métier de papetier dans

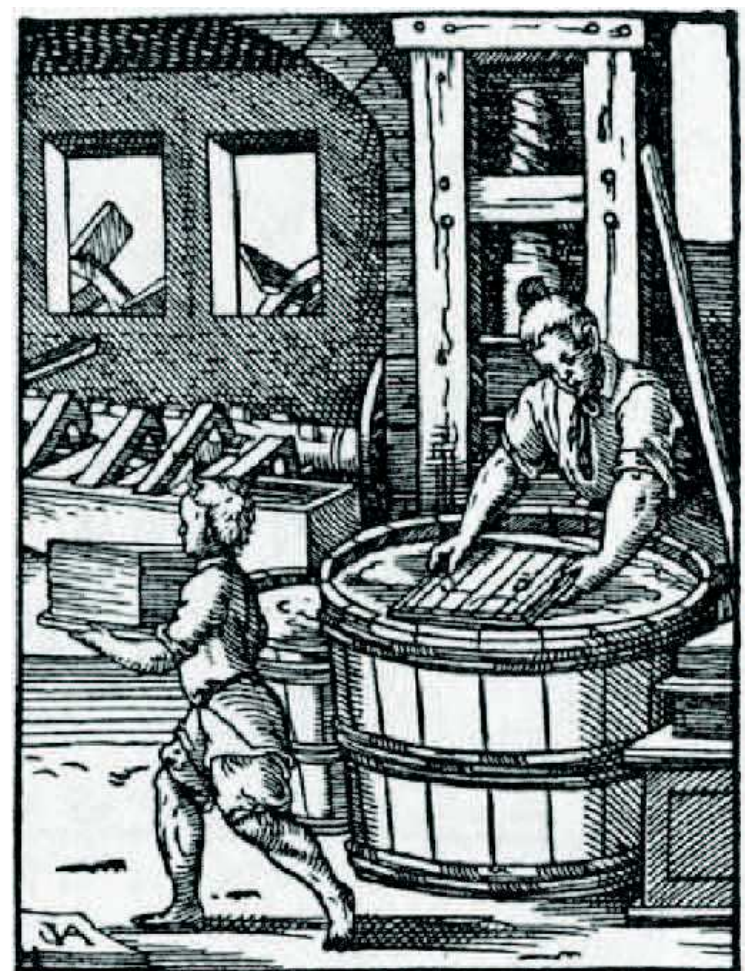

Fig. 2 : Le papetier " dans Jost Amman, Livre des métiers (Ständebuch), Francfort-sur-le-Main, 1568 (sur la bibliothèque numérique d'histoire sociale et économique de Cologne : http://www.digitalis.uni-koeln.de/).

son ensemble, et non d'expliquer la chaîne opératoire. Concernant les machines cependant, la fonction testimoniale est le plus souvent cumulée avec une fonction explicative, ce qui explique notamment l'absence de telles contradictions dans les théâtres de machines.

\section{La fonction explicative}

Dans l'esprit des historiens, et sans doute ne sontils pas les seuls, le texte tend, aujourd'hui encore, à monopoliser la fonction explicative des documents, les images étant soit cantonnées à un rôle illustratif, soit considérées si trompeuses qu'elles nécessitent des explications particulières. Non qu'on cherche ici à remet- 
tre en cause toute herméneutique de l'image - bien au contraire - mais on pense en revanche qu'elles peuvent avoir été conçues, non pour illustrer un texte, mais pour expliquer une idée, un concept, ou encore le fonctionnement d'un objet. Les manuels de montage Ikea sont d'ailleurs la preuve qu'il est possible de concevoir des images qui assurent en elle-même une fonction explicative. Dans les images de machines de l'époque moderne, cette fonction a été majeure, pour ne pas dire majoritaire. Qu'elles se veulent pédagogiques ou traces d'autres inventions, privées ou destinées à un large public, ces images ont des caractéristiques communes qu'il ne faut pas négliger.

Concernant les machines, la fonction explicative se résume le plus souvent à chercher à en montrer le fonctionnement, et à placer, à utiliser des techniques graphiques qui tendent le plus souvent à extraire de la machine concrète, réaliste, les caractéristiques principales, les principes. Parmi ces techniques, la généralisation aux différentes parties de la machine, de la cutaway view, technique qui consiste à « découper et retirer » des morceaux de la machine (souvent du bâti) afin de dévoiler ce qu'il y a derrière, permet de guider le regard vers des mécanismes particuliers et d'attirer ainsi l'attention sur ce qui permet le fonctionnement de la machine. Cependant, cette technique est loin d'être la seule, et la principale difficulté des nouveaux modes de représentation réside dans le fait qu'ils nécessitent la mise en place d'un code graphique commun, afin que les procédés employés soient bien compris par tous les destinataires de l'image. Cela explique d'abord la très grande stabilité de l'usage de la perspective ${ }^{7}$. Même si schémas et éclatés accompagnent de plus en plus souvent l'iconographie mécanique, il est rare, jusqu'au XIX siècle, de voir, de façon isolée et comme unique référence explicative, des schémas de machine. Les images du traité des machines de Jean-Nicolas-Pierre Hachette fait encore appel à de telles représentations, qui cumulent fonction testimoniale et explicative. C'est pourquoi il est intéressant de faire l'histoire de l'insertion progressive de certaines techniques graphiques dans les images de machines destinées au public, étant entendu que la plupart des ouvrages personnels usent de codes propres à l'auteur et que celui-ci n'a pas à se soucier de leur compréhension par d'autres. Sans qu'il soit possible ici de faire une description - et a fortiori une histoire des multiples techniques graphiques et de donner à voir la complexité de leurs multiples combinaisons, on se contente de mettre en avant les deux techniques les plus utilisés : l'éclaté et le schéma ${ }^{8}$.

\section{Détailler la machine : l'éclaté}

L'éclaté d'abord, qui consiste à isoler une partie d'un tout et à le placer ailleurs sur la page, est un procédé qui semble apparaître, en ce qui concerne les machines, au début du XV $\mathrm{XV}^{\mathrm{e}}$ siècle, et notamment dans les œuvres de Mariano Taccola et Giovanni Fontana, si on en croit la $\mathrm{DMD}^{9}$. Cette technique est loin d'être évidente dans un monde où domine une pensée aristotélicienne pour qui « la machine [...] constituait un tout que l'on ne peut ni séparer, ni analyser et encore moins calculer. ${ }^{10}$. En mécanique, il est d'ailleurs rare que les éclatés concernent des pièces seules, il s'agit le plus souvent de mettre en avant une relation entre deux organes de la machine, pour faciliter la compréhension du mécanisme central, ou de celui qui apporte justement un peu de nouveauté. Ainsi, les premiers éclatés apparaissent le plus souvent dans un espace vide de la feuille, sans place particulière ni séparation de l'espace de la représentation globale de la machine et celui des détails mis en avant. Le code graphique de l'imprimé évolue alors dans plusieurs directions, développées simultanément à partir du XVI siècle, et qui révèlent toutes les tensions qui se font jour entre les fonctions testimoniales et explicatives des images imprimées et destinées à un plus large public.

Surtout visible dans l'imprimé du fait de sa facilité de lecture dans un monde où les images deviennent de plus en plus réalistes ${ }^{11}$, la première évolution de l'éclaté vise à donner au mécanisme mis en avant une place particulière et ancrée dans le réel sur la page. Ainsi, dans le Ramelli ou même dans le De Re Metallica, les auteurs des images préfèrent placer leurs éclatés posés sur le sol, avec une ombre portée. Parfois même, quand les organes utilisés sont radicalement nouveaux, comme dans 
le cas des pompes rotatives de Ramelli (voir figure 1), les pièces sont démontées sur une surface de manière à montrer comment est fabriquée la pièce. Loin d'être distincte de la fonction testimoniale, l'explication visuelle apportée ici vient en continuité d'un témoignage global, que l'éclaté prolonge dans le détail, comme le faisait la « cutaway view ». En pointant ce qui est nouveau, ces techniques visent à donner au lecteur des clés de lecture plus précises qui lui permettent à la fois d'apprécier la réalité de l'existence de tel ou tel mécanisme, et de lui enlever par là même son caractère inquiétant en rendant compte en détail de son fonctionnement.

La seconde forme d'éclaté, relativement rare, est surtout développée dans le livre de Besson. Le parti formel est exactement l'inverse du précédent, non que la représentation de l'objet soit moins réaliste en elle-même, mais l'éclaté couvre ici toute la surface de la page, et permet de lire la composition de l'objet pas à pas, et même pièce par pièce en utilisant l'unité de construction la plus basse. Le sens de lecture se fait alors de haut en bas, de l'intérieur vers l'extérieur, du plus simple vers le plus composé. L'auteur use de cette technique graphique principalement pour les instruments mathématiques. Cela permet non seulement, et d'une tout autre manière, de guider le regard et de mettre en avant les détails de l'instrument, mais encore de comprendre la façon dont il est monté. Assez peu repris pendant la période moderne, cette forme d'éclaté de montage peut être considérée comme le témoin d'essais qui n'ont pas trouvé un public capable d'en comprendre les codes.

Une troisième forme d'éclaté se développe : il s'agit conjointement de placer dans le vide les mécanismes importants de la machine, tout en stylisant fortement le dessin, c'est-à-dire en supprimant les ombrages, en diminuant le réalisme du rendu de l'épaisseur des différents organes mécaniques ou du bâti. Dérivée sans doute à la fois de la technique habituelle de l'éclaté et d'une représentation plus grossière, cette forme, que l'on peut nommer éclaté « de principe » conduit, au cours du XVII ${ }^{\mathrm{e}}$ siècle, vers la forme la plus canonique des images de machines à fonction principalement explicative : le schéma.

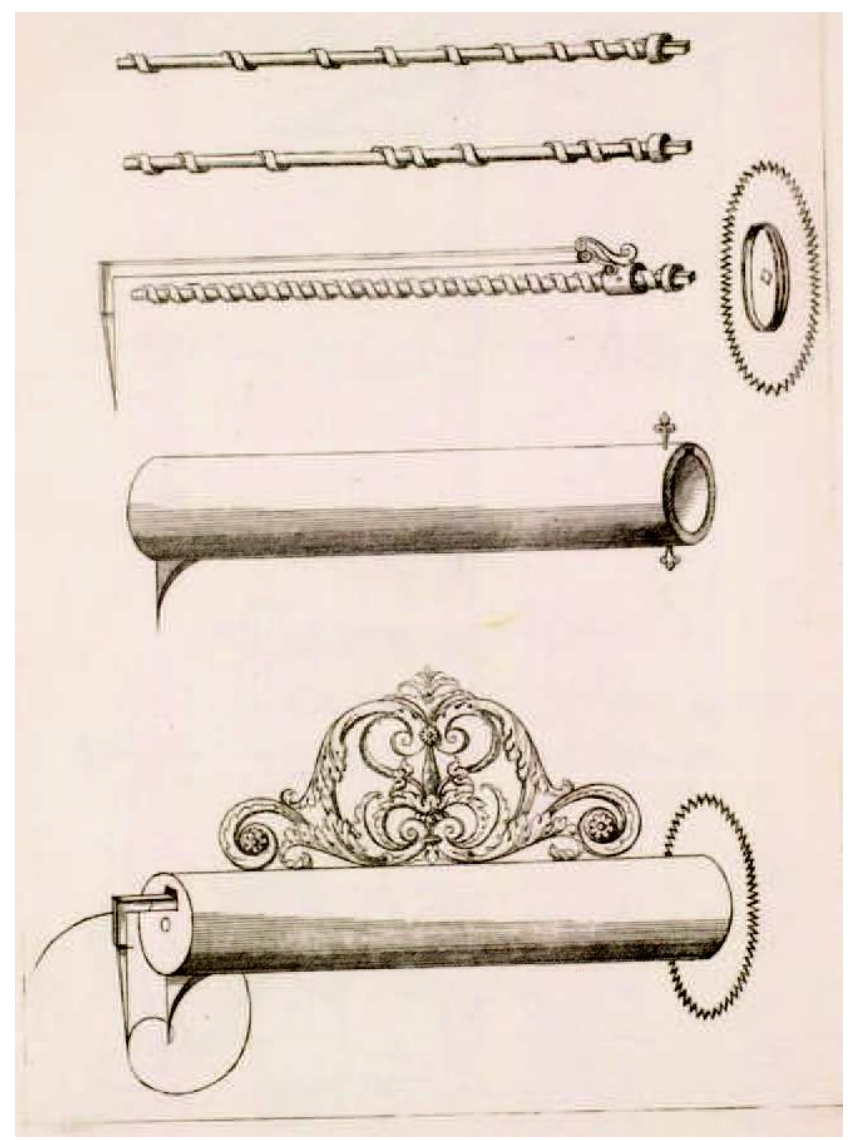

Fig. 3 : Planche 6 de BESSON Jacques, Instrumentorum et machinarum [...] liber primus, Paris, chez Fleury Prevost, vers 1571 (Gallica, BNF).

\section{Visualiser l'organisation : le schéma}

Le schéma est un mode de représentation graphique aujourd'hui courant et qui ne pose souvent à nos contemporains qu'assez peu de problèmes de lecture. Les schémas ne sont pas des dessins réalistes, en ce qu'ils ne rendent compte de la plupart des informations matérielles qu'avec l'usage de certains symboles, et jamais de façon directe. En cela, ils ne sont pas appréhendables sans une certaine connaissance des symboles utilisés, nécessitant parfois un véritable apprentissage, comme pour les schémas électriques contemporains. Le 
schéma est un outil à la fois de conception et d'explication très puissant : il permet de focaliser l'attention sur tel ou tel type d'information, en faisant abstraction de celles sur lesquelles l'auteur ne souhaite pas s'interroger. Dans les machines, on l'a dit, les images explicatives ont surtout vocation à faire saisir au lecteur le fonctionnement de la machine, ou autrement dit les interactions entre ses différentes parties. Pour cela, le schéma offre un outil intéressant en ce qu'il permet de faire abstraction d'une série d'informations matérielles (spatialité, matériaux, formes exactes) et de se concentrer sur les mécanismes et leur disposition.
Les premiers schémas sont utilisés dans des ouvrages théoriques et mathématiques, et il s'agit souvent moins de montrer la constitution d'un objet que d'inscrire les mouvements de ses parties dans des figures géométriques, et d'aider ainsi à poser certains calculs. Cependant, le schéma n'a pas émergé en une seule fois, il semble plutôt le fait d'une maturation et d'une abstraction successive des informations matérielles. D'une certaine façon, il a toujours existé une certaine forme de schéma dans les images dans lesquelles la fonction explicative prédomine largement, comme c'est par exemple le cas des gravures sur bois représentant des

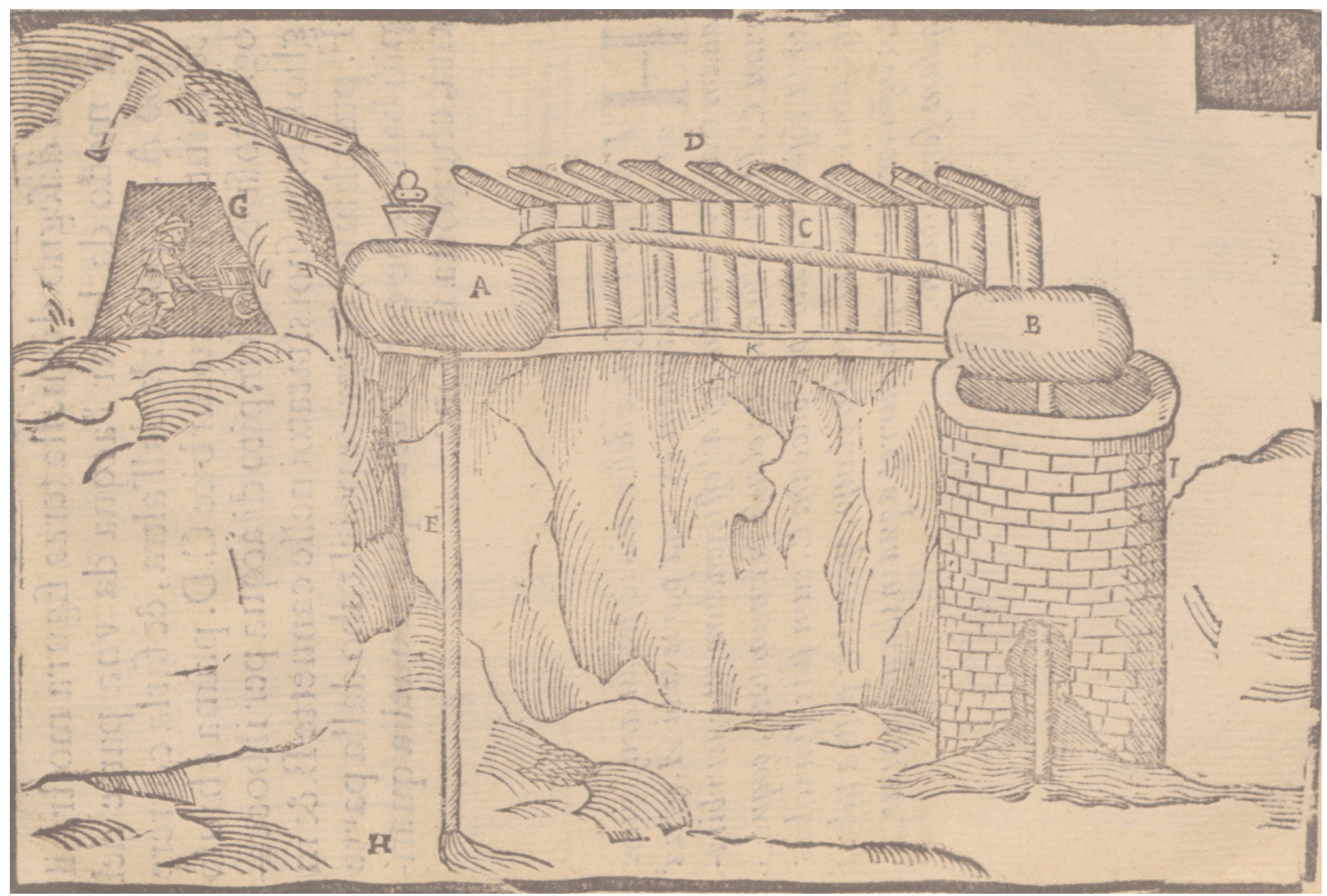

Fig. 4 : Planche 2 de BRANCA Giovanni, Le Machine, Rome, chez Jacomo Mascardi (impr.) à la demande de Jacomo Marcuci (éd.), 1629 (Base « Kinematic Models for Design » de l'Université Cornell : http://kmoddl.library.cornell.edu). 
machines hydrauliques que Giovanni Branca, à la demande son protecteur, commente dans Le Machine ${ }^{12}$. Celles-ci ne sont ni réalistes, ni schématiques à proprement parler, elles sont simplement grossières. Non seulement la maçonnerie et la texture des matériaux ne sont que très rarement rendues, mais les objets sont souvent démesurés par rapport à l'environnement représenté, et leur spatialité n'est pas respectée. Parfois même, les objets ne sont pas à l'échelle les uns par rapport aux autres, afin de mettre l'accent sur leurs relations. De la même manière, certaines roues à eau sont représentées de façon très stylisée, et la plupart des

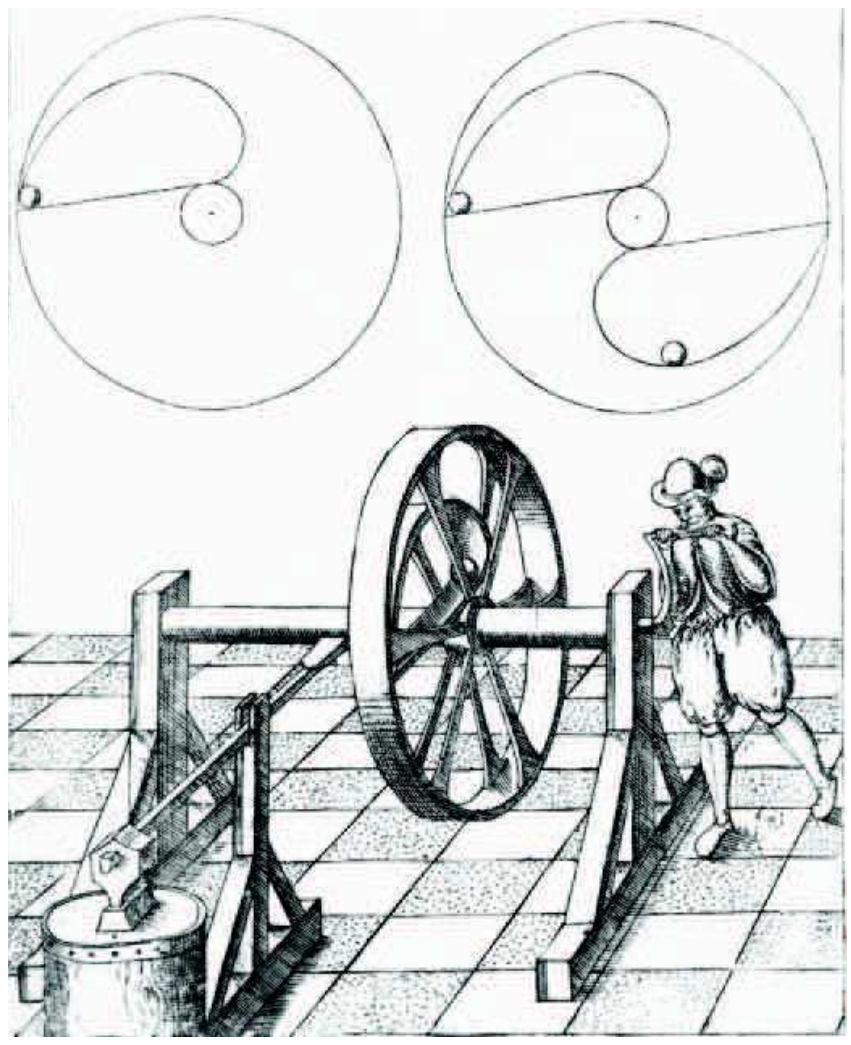

Fig. 5 : Planche 27 de ERRARD DE BAR LE DUC Jean, Le premier livre des instruments mathématiques méchaniques, Nancy, chez Jan Janson, 1584 (tiré de Wolfgang Lefêre et Marcus Popplow, Database Machine Drawings, MPIWG : http://dmd.mpiwg-berlin.mpg.de).

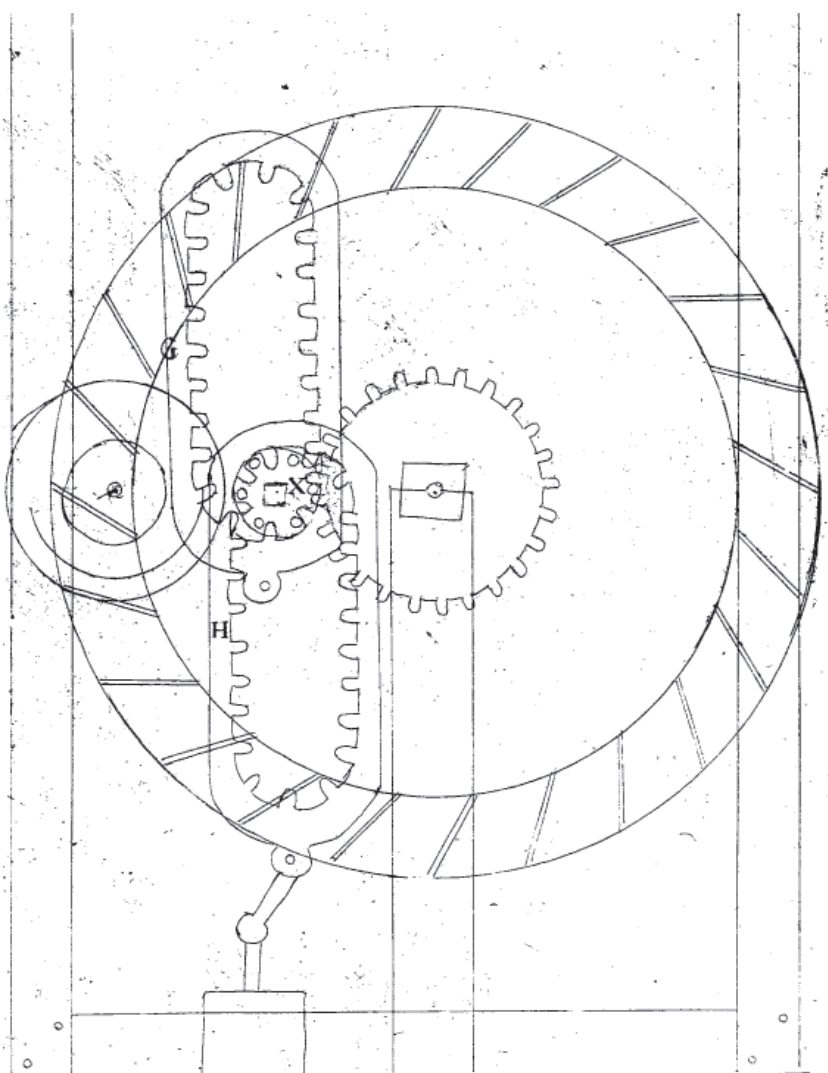

Fig. 6 : Planche 15 CAUS Salomon de, Les raisons des forces mouvantes, Francfort, J. Norton, 1615 (Conservatoire national des arts et métiers, Conservatoire numérique http://cnum.cnam.fr).

tuyaux, normalement enterrés sont représentés superposés aux montagnes dessinées à l'arrière-plan. C'est qu'il s'agit ici de suggérer, plus que de représenter, l'ancrage dans le réel des machines ; l'objectif principal étant de montrer l'organisation du dispositif.

Ce type de représentation est ensuite repris, mais souvent en éclaté. Sortant un dispositif mécanique de sa gangue réaliste, l'auteur place, à côté de la machine représentée en perspective et en contexte, une machine aux traits simplifiés, avec seulement les pièces les plus importantes représentées en perspective. Cela permet 
là encore de guider le regard vers ce qui est important et/ou nouveau dans l'invention présentée, comme dans les théâtres de Jacques Besson ou de Jean Errard, ou dans certaines représentations du De Re Metallica. C'est sans doute dans cet espace dévolu à l'éclaté que le schéma peut le plus facilement se développer. En effet, à côté d'une représentation à l'époque plus conventionnelle et plus compréhensible, les auteurs peuvent expérimenter plusieurs codes graphiques, et notamment l'insertion dans le schéma de pointillés ou de traits fins visant à montrer les mouvements à effectuer sur la machine, les flèches étant assez rares. Le meilleur exemple de cette cohabitation féconde entre schéma et représentation réaliste est sans aucun doute donné par Salomon de Caus qui, sur trois machines, donne d'abord la représentation d'une machine en perspective et, dans une seconde planche, « à cause que la precedente machine est fort difficile à entendre », il place une " orthographie », c'est-à-dire ni plus ni moins qu'une vue de côté, ou une « ignographie » (plan vu de haut) comme on en trouve aujourd'hui dans tous les dessins techniques issus des bureaux d'études.

\section{Reproduire, ou concevoir ?}

Ce qu'il est convenu aujourd'hui d'appeler le graphisme technique, ces dessins visant à la communication entre le bureau d'étude et la fabrication, apparaît plutôt tardivement en ce qui concerne les machines. Comme le constate en effet Yves Deforge ${ }^{13}$ puis Bernard Quéré $^{14}$ et Wolfgang Lefèvre ${ }^{15}$, rares sont les dessins de l'époque moderne qui puissent être considérés comme des blueprints, ces dessins servant à la communication entre un concepteur et un fabriquant. La chose ne manque pas d'étonner si l'on considère les grandes réalisations mécaniques de l'époque moderne, comme la machine de Marly, mais, si les méthodes et le complexe technique de la fabrication des machines a été assez peu étudié, il y a fort à parier que, comme Éric Rieth l'a montré en étudiant l'augmentation du nombre de pièces gabariées sur les membrures d'un navire ${ }^{16}$, la conception précise des pièces d'une machine ne nécessite pas obligatoirement l'emploi systématique de dessins, cer- tains savoir-faire permettant de réaliser des gabarits directement à échelle $1 / 1$, à l'aide d'instruments particuliers. De fait, le dessin technique de reproduction, tel que nous le connaissons, est principalement lié au modèle du bureau d'étude, dans lequel la conception est plurielle et la communication entre le concepteur et le fabriquant moins directe, davantage qu'à la complexité des objets. Aussi notre étude, limitée à l'époque moderne et focalisée sur les images d'usage publique ne reviendra pas sur ces techniques iconographiques permettant la reproduction

Ceci dit, plusieurs besoins liés à la conception des machines poussent les ingénieurs de l'époque moderne à utiliser d'autres méthodes de représentations. Le premier ce ces besoins est sans doute la possibilité de disposer, dans son esprit, d'un certain nombre de machines et de mécanismes qui puissent être mobilisés dans un contexte précis ; une sorte de catalogue dont certaines caractéristiques seraient précisées pour aider le concepteur à se faire une idée plus précise des possibilités et des besoins en matière première et en main d'œuvre. Le second besoin émerge avec la science du XVIII ${ }^{\mathrm{e}}$ siècle et accompagne la nécessité de plus en plus grandissante de calculer les machines. Il faut alors pouvoir disposer de modes graphiques facilitant le calcul et donc, un troisième besoin, le dimensionnement des pièces et des mécanismes. Ce dimensionnement demande l'apprentissage de techniques permettant de dessiner avec précision taille et forme des différents organes mécaniques utilisés. Ces dessins sont sans doute les plus proches des dessins techniques d'aujourd'hui, destiné à la production par un tiers des pièces représentées.

\section{Vers la constitution d'un catalogue}

Le besoin de disposer d'un catalogue pousse d'une certaine façon à l'extension de la fonction testimoniale : il s'agit en effet de mieux se rendre compte de ce que suppose la construction de telle ou telle machine, non seulement en terme de fonctionnement, mais aussi en terme de composition et d'occupation de l'espace par exemple. La plupart de ces informations plus précises 
sont apportées dans le texte : nombre de dents d'une roue, de fuseaux d'une lanterne, dimensions des roues, des caisses et des différentes pièces sont ainsi de plus en plus précisés. Il faut insister sur ce point, car nous sommes face ici à l'exact contraire de notre vision contemporaine, dans laquelle le dessin permet d'obtenir des informations précises de dimensionnement et où le texte revient sur l'explication et le calcul. Cependant, chez certains auteurs, et notamment ceux qui décrivent des machines déjà existantes, certains outils graphiques commencent à être développés.

Le livre de Vittorio Zonca ${ }^{17}$ est à cet égard exemplaire. D'abord, il propose un quatrième mode d'éclaté, plaçant dans un espace à part, bien délimité, les pièces nécessaires au bon fonctionnement des engrenages et donc de la transmission du mouvement. Il ne s'agit clairement plus de montrer le fonctionnement de la machine, par ailleurs expliqué dans le texte, mais bien de mettre à part la pièce pour permettre au lecteur de la percevoir en elle-même, séparément, et de ne pas penser qu'un engrenage puisse être composé d'une seule pièce. C'est une façon de désigner la pièce, d'attirer le regard vers elle et d'amener le lecteur à en regarder les caractéristiques avec plus d'insistance, notamment le nombre et la disposition régulières des dents et des fuseaux, ou la construction de la roue. Zonca accompagne par ailleurs cette légende visuelle d'une véritable légende écrite, à part du texte d'explication, qui n'apparaît jamais dans les autres théâtres de machines.

Le Novo teatro insère aussi une échelle dans certaines de ces images, pour en préciser la dimension et donc l'occupation spatiale, de même qu'il est le premier à utiliser plusieurs vues d'une même machine pour permettre au lecteur un meilleur confort dans l'appréhension mentale de la machine, dans une planche qui est sans doute celle qui se rapproche le plus d'un dessin technique contemporain, à ceci près que la machine à faire du fil de plomb est ici ouverte en deux parties, sans que les deux pièces ne soient représentées sous tous leurs angles ${ }^{18}$.

Quoiqu'il en soit, on remarque dans les images du Zonca que l'insertion de ces techniques graphiques ne supprime jamais une représentation en perspective et en contexte de la machine. C'est que la fonction testimoniale de l'image répond aussi en grande partie à ce besoin de catalogage précis, en permettant à un œil non habitué de saisir d'un seul regard la taille, la spatialité et la complexité de la machine. Le même type d'image, mêlant schémas, graphiques et représentations en perspective reste omniprésent dans les ouvrages théoriques du XVIII ${ }^{\mathrm{e}}$ siècle.

Des dessins pour calculer, des dessins pour dessiner

La somme de Bélidor paraît en 1737 ; celle de Leupold dès 1724 ; et durant le XVIII ${ }^{\mathrm{e}}$ siècle se multiplient les livres hollandais sur les moulins à vent. On remarque toujours, dans ces ouvrages, une forte similarité du graphisme et du vocabulaire formel, depuis les images de machines en perspective et en contexte, jusqu'aux schémas d'ensemble ou les figures plus géométriques. Reprendre l'ensemble des techniques graphiques rencontrées et en faire la généalogie mériterait sans doute un article complet, mais il est possible ici de mentionner deux types de représentations.

Le premier type rassemble des représentations schématiques : il s'agit très clairement de faciliter au lecteur la compréhension d'une expérience et d'un calcul. L'image consiste alors à placer une figure géométrique, possédant des séries de mesures, à côté d'une image un peu plus réaliste (coupe, vue en perspective, etc.), la figure géométrique servant de guide pour le dessin ou la conception d'une pièce similaire, le calcul en lui-même et l'explication de la façon d'user du dessin résidant quant à lui dans le texte. Pour autant, si le calcul en luimême réside dans le texte, l'image n'en demeure pas moins d'une importance capitale, en ce qu'elle ancre dans la vision du lecteur un mode graphique de représentation de la machine selon les règles de la Statique, développée notamment par Simon Stevin ou Christian Wolff. Il s'agit là d'aider l'ingénieur à schématiser une machine pour lui permettre d'en calculer l'efficience par la suite, mais aussi à en permettre le dimensionnement, et donc un tracé personnalisé en fonction des besoins du terrain. 


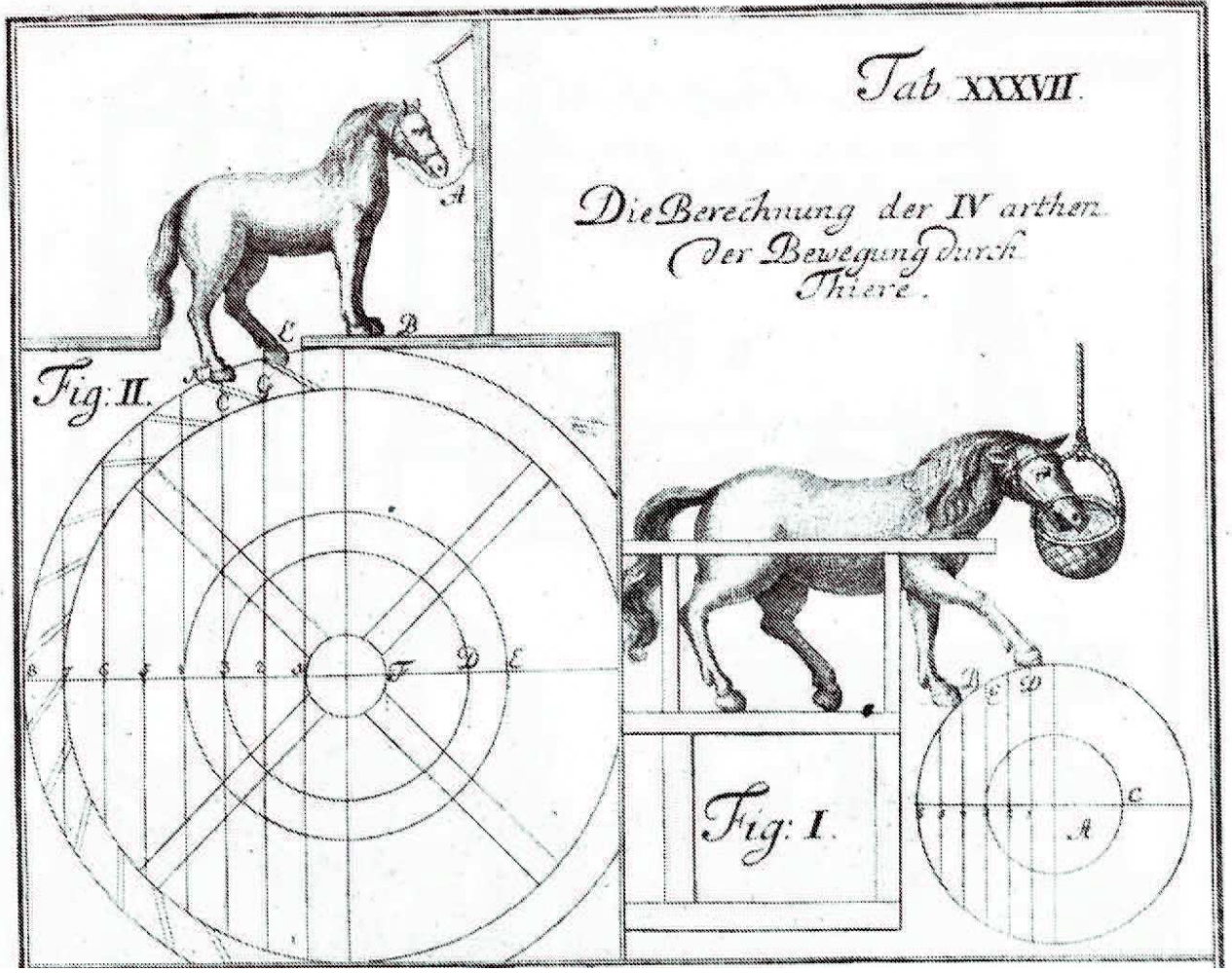

Fig. 7 : Planche 37 de LEUPOLD Jacob, Theatrum machinarum generale, Leipzig, 1724: "Calcul du quatrième type de mouvement par un animal » (Base "Kinematic Models for Design » de l’Université Cornell : http://kmoddl.library.cornell.edu).

Le second type de représentation est davantage visible dans les ouvrages plus spécialisés, comme celui de Bélidor, ou ceux édités à Amsterdam par Pierre Schenck. Il s'agit là en fait d'une autre tradition que celle des images de machines, et qui vient plus probablement de l'architecture. Le titre même de l'ouvrage de Bélidor, L'Architecture hydraulique, et le fait que, dès la Renaissance, la machine soit considérée comme un bâtiment en mouvement (Salomon de Caus), permet d'ailleurs de comprendre que certaines techniques graphiques, et sans doute celles qui vont le plus directement permettre l'émergence du graphisme technique soient issues de l'architecture. Cette évolution reste encore largement à explorer.

\section{Conclusion}

La complexité et les emprunts successifs de techniques graphiques, d'une période à l'autre, rendent difficile une séparation nette entre les différentes fonctions des images. On aura donc voulu, par cet essai de typologie, souligner l'intérêt d'une approche par fonctions cumulatives. Une même image, comme un texte d'ailleurs, peut répondre à plusieurs objectifs. Et si cette pluralité fonctionnelle tend à disparaître de nos jours, elle a peut-être été la règle hier. Entendons-nous bien, en souhaitant ouvrir le lecteur à la question de la transformation du but recherché par l'auteur en une fonction générale de l'image et à celle du cumul possible de ces fonctions, il n'était pas question ici d'affirmer que cette 
typologie épuise à elle seule le sens de l'image. L'analyse esthétique des images, comme la nécessité de rendre compte des objectifs de l'auteur et de bien contextualiser les images, demeurent nécessaires à une compréhension plus complète des images.

Mais je voudrais revenir sur ce que cette étude a pu nous apprendre sur les raisons de l'émergence progressive du dessin industriel, de type blueprint. Nous avons vu qu'en terme de dessins de machines, la fonction testimoniale prenait racine dans la nécessité pour les ingénieurs à la fois de faire valoir leur ingenium et d'apprivoiser l'innovation. Cette fonction était somme toute majeure, et dominait les autres, au point d'imposer un minimum de mise en scène et la perspective comme horizon de tout dessin de machine. Cependant, petit à petit, la fonction explicative des images semble prendre le pas, dans les livres imprimés, sur la fonction testimoniale. La mise en place d'un statut d'ingénieur plus clair, l'installation de ce nouveau corps dans le paysage social, et la multiplication des modèles, contribuent à ce mouvement, qui se traduit notamment par la publication d'ouvrages de plus en plus théoriques, dans lesquels le schéma constitue une sorte de paradigme graphique concurrent de celui de la perspective et de la mise en contexte.

Cette concurrence se voit très bien dans les méthodes de représentations du caché. Éclaté en contexte et cutaway view ont l'avantage de combiner à la fois une fonction purement monstrative et explicative, par l'usage d'un graphisme réaliste et une continuité intellectuelle avec le mode de représentation commun à tous à l'époque : la perspective. Cela explique leur place dominante dans les techniques graphiques de représentation des machines du XVI ${ }^{\mathrm{e}}$ à la fin du $\mathrm{XVIII}^{\mathrm{e}}$ siècle. De même, les représentations grossières et les points de vue multiples cumulent aussi ces avantages, quoiqu'ils soient sans doute moins instinctivement accessibles, du fait de leur manque de réalisme. Ce hiatus avec les règles des bonnes proportions et de la perspective accélère leur disparition à la fin de la Renaissance, alors que le schéma se développe.

La plupart des outils graphiques permettant d'amé- liorer la fonction explicative des images, comme les premières représentations schématiques, les éclatés de montage, les vues par transparence ou encore les pointillés, sont des outils graphiques qui n'ont que peu de place dans une représentation très réaliste des machines, et ils rentrent en concurrence directe avec les autres outils de représentation de ce paradigme testimonial que sont la cutaway view et l'éclaté en contexte. Ces outils trouvent en revanche à se développer avec l'émergence du schéma comme nouveau paradigme de représentation.

Ce nouveau paradigme, qui intègre beaucoup plus facilement les autres outils graphiques, s'installe dans les images des techniques au fur et à mesure que les traités se font plus pédagogiques, plus explicatifs. Et, bien que la perspective demeure utilisée, les proportions et les échelles ne sont pas toujours respectées et la fonction testimoniale, toujours présente ne serait-ce que « pour se faire une idée », cède le haut du pavé aux séries de techniques qui permettent de décortiquer la machine, de l'analyser.

À partir de ce paradigme du schéma peut se mettre en place, sans doute à partir du XIX ${ }^{\mathrm{e}}$ siècle, une grammaire commune du dessin de machine, qui permettra plus tard le développement des techniques de conception et de reproduction par une généralisation de ces techniques graphiques à toutes les pièces et toutes les techniques. D'une certaine façon, la machine est devenue si bien expliquée qu'elle en est devenue reproductible ou re-conceptualisable.

\footnotetext{
${ }^{1}$ Voir notamment LEFEVRE Wolfgang (éd.), Picturing Machines : 1400-170o, Cambridge, MIT Press, 2004 ; DEFORGE Yves, Le graphisme technique : son histoire et son enseignement, Seyssel, Champ Vallon, 1981; et QUERE Bernard, La communication scientifique et technique par les outils graphiques de 1750 à 1850 dans le contexte de la Bretagne, thèse dirigée par Jean Dhombres et soutenue à l'EPHE le 15 décembre 2005. La thèse est disponible librement sur HAL-SHS.

${ }^{2}$ Malgré de nombreuses études, le dessin de machines pose toujours questions : voir note précédente, notamment le chapitre de DEFORGE, 1981, op. cit., chapitre : « Les machines, enfin », pp. 6375, et QUERE, op. cit., chapitre 2, 3 et 6, pp. 86-174 et 269-305.
} 
Transition : tentatives de représentations no uvelles développant la fonction explic ative

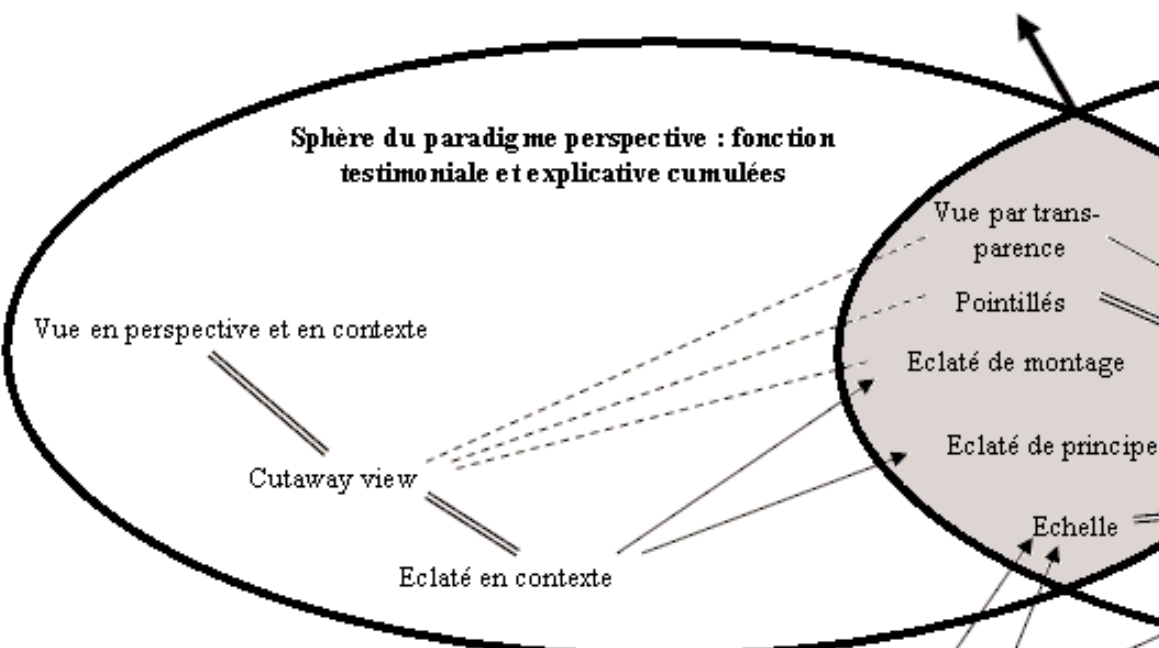

Fig. 8 : Schéma de l'évolution des techniques graphiques

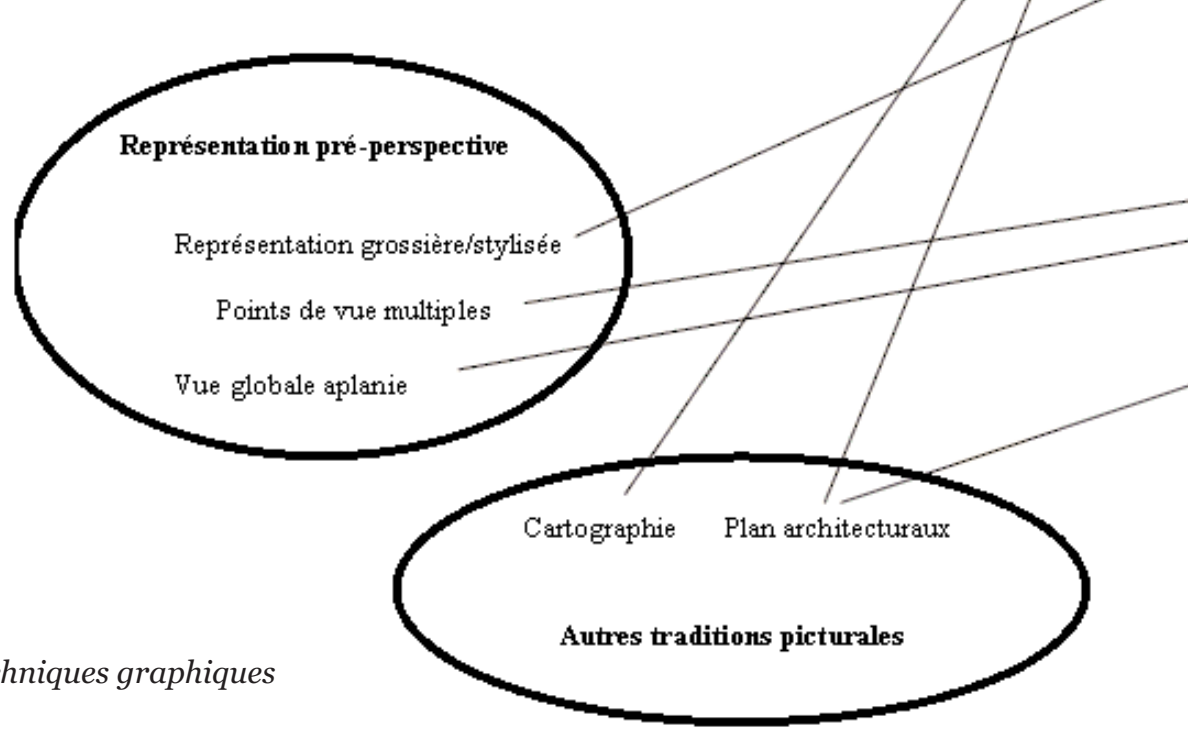




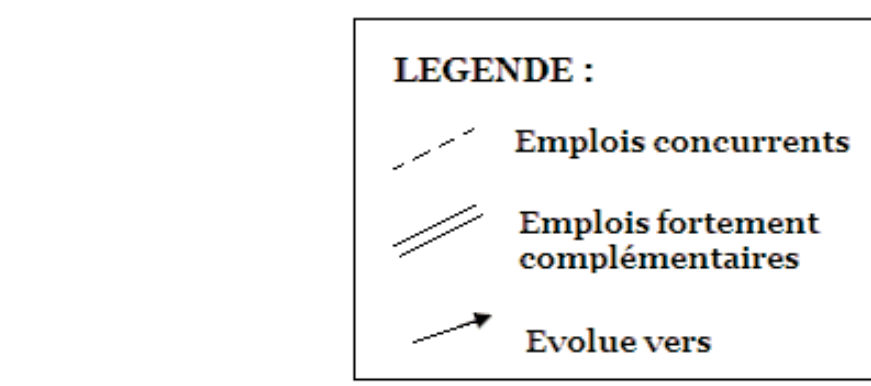

Sphère du paradig me schématique : indépendance de la fonction explicati-

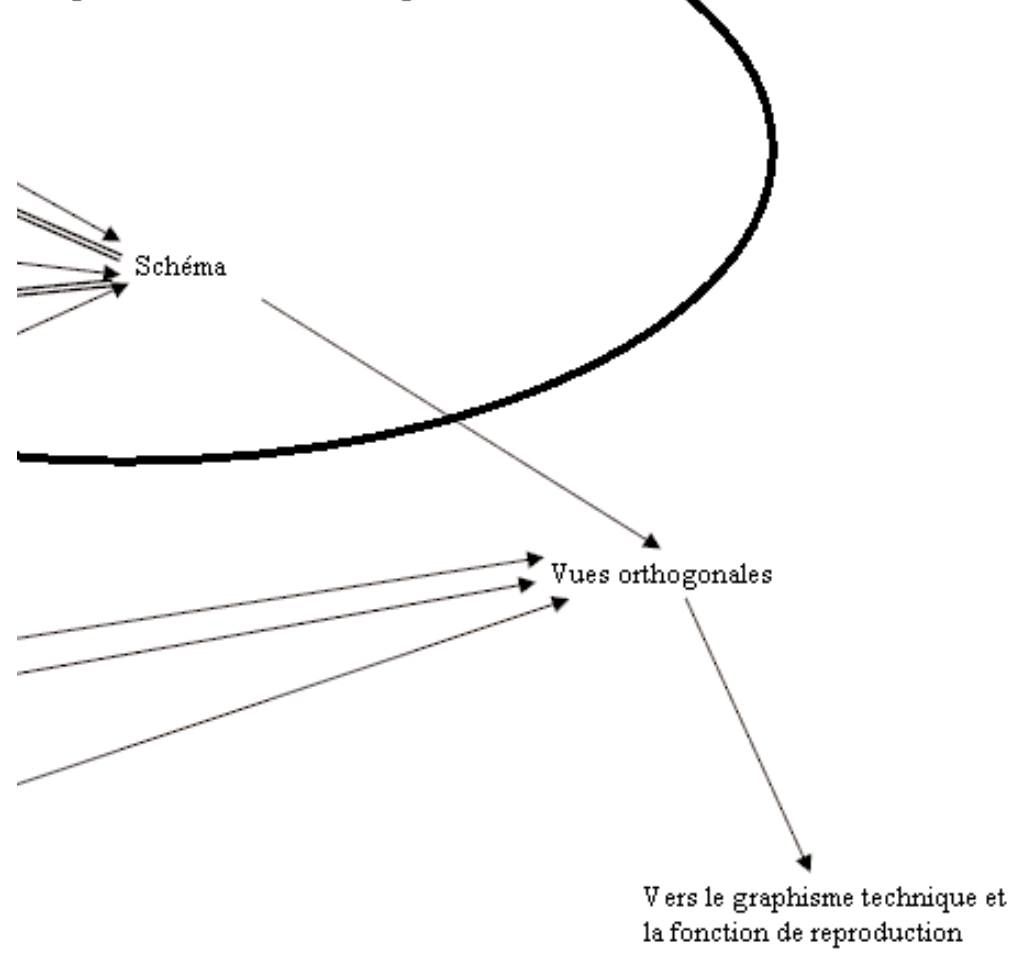


${ }^{3}$ SHAPIN Steven, « Une pompe de circonstance. La technologie littéraire de Boyle ", Culture technique, 14, 1985, p. 77.

${ }^{4}$ GARÇON Anne-Françoise, Entre l'État et l'usine : l'école des mines de Saint-Étienne au XIX ${ }^{e}$ siècle, Rennes, PU-Rennes, 2004, chapitre 5 ; GARÇON Anne-Françoise, " La science, l'esthétique et l'éculé », dans HATCHUEL Armand et WEIL Benoît (dir.), Les nouveaux régimes de la conception, Cerisy-la-salle, Vuibert et Centre culturel international de Cerisy, 2008. Yves Deforge avance une idée similaire.

${ }^{5}$ Sur la permanence de ce motif au XVIIIe siècle, voir PIERROT Nicolas, "Visites de manufactures ", dans PIERROT Nicolas, Les images de l'industrie en France. Peintures, dessins, estampes, 17601870, thèse de doctorat d'histoire dirigée par Anne-Françoise Garçon, Université Paris 1 Panthéon-Sorbonne, Centre d'Histoire des Techniques (CH2ST-EA 127), 2010, vol. 1, p. 255-276.

${ }^{6}$ AMMAN Jost, Eygentliche Beschreibung aller Stände auff Erden, Francfurt-sur-le-Main, chez Hans Sachs, $1568 .{ }^{7}$ Notons, à l'inverse, que ce qui est évident n'est pas expliqué, ni parfois représenté.

${ }^{8}$ Sur l'importance de la fonction explicative dans l'émergence du graphisme technique, voir Quéré Bernard, 2005, op.cit., S'il n'utilise pas les mêmes mots, Bernard Quere montre plusieurs exemples qui viennent confirmer le poids des productions à visée pédagogique dans la mise en place de codes graphiques techniques. Par ailleurs, il commence lui aussi par mettre en évidence l'héritage de la perspective dans des carnets uniquement descriptifs.

${ }^{9}$ LEFEVRE Wolfgang et POPPLOW Marcus, Database Machines Drawings, http://dmd.mpiwg-berlin.mpg.de/home, dernière mise à jour janvier 2009. Recensant des images de machines du XIII ${ }^{\mathrm{e}}$ au XVII siècle, la base ne montre aucun éclaté pour les œuvres de Guy de Vigevano, de Villard de Honnecourt, ou de Conrad Kyeser.

${ }^{10}$ DEFORGE, 1981, op. cit.

${ }^{11}$ IVINS William M., " La rationalisation du regard », Culture Technique, 14, juin 1985, pp. 30-37.

12 BRANCA Giovanni, Le Machine, Rome, chez Jacomo Mascardi (impr.) à la demande de Jacomo Marcuci (éd.), 1629. Voir sur ce point RAVIER Benjamin, Le discours technique dans les théâtres de machines (1572-1629), mémoire de master réalisé sous la direction de GARÇON Anne-Françoise, Université Paris 1 Panthéon-Sorbonne (EA 127-CH2ST), 2009, p. 147-151.

${ }^{13}$ DEFORGE, 1981, op. cit., p. 63.

${ }^{14}$ QUERE, 2005, op.cit.

${ }^{15}$ LEFEVRE Wolgang, «The Emergence of Combined Orthographic Projections », dans LEFEVRE, 2004, op. cit.

${ }^{16}$ RIETH Eric, Le maître-gabarit, la tablette et le trébuchet, essai sur la conception non-graphique des carènes du Moyen Âge au XX siècle, Paris, CHTS, 1996.

${ }^{17}$ ZONCA Vittorio, Novo teatro di machine et edificii per varie et sicure operationi, Padoue, chez P. Bertelli, 1607. Disponible sur le site Kinematic Models for Design de l'Université Cornell : http://kmoddl.library.cornell.edu/ (consulté en octobre 2012).
${ }^{18}$ Il s'agit de la planche située entre la page 79 et la page 80 de ZONCA, 1607, op. cit. 